\title{
Application of novel analytical ultracentrifuge analysis to solutions of fungal mannans
}

\author{
Richard B. Gillis ${ }^{1,2}$ • Gary G. Adams ${ }^{1,2}$ • David T. M. Besong ${ }^{3}$ Eva Machová ${ }^{4}$. \\ Anna Ebringerová $^{4} \cdot$ Arthur J. Rowe $^{2} \cdot$ Stephen E. Harding $^{2} \cdot$ Trushar R. Patel $^{5}$
}

Received: 25 May 2016 / Revised: 8 July 2016 / Accepted: 11 July 2016 / Published online: 21 July 2016

(C) The Author(s) 2016. This article is published with open access at Springerlink.com

\begin{abstract}
Polysaccharides, the most abundant biopolymers, are required for a host of activities in lower organisms, animals, and plants. Their solution characterization is challenging due to their complex shape, heterogeneity, and size. Here, recently developed data analysis approaches were applied for traditional sedimentation equilibrium and velocity methods in order to investigate the molar mass distribution(s) of a subtype of polysaccharide, namely, mannans from four Candida spp. The molecular weight distributions of these mannans were studied using two recently developed equilibrium approaches: SEDFITMSTAR and MULTISIG, resulting in corroboratory distribution profiles. Additionally, sedimentation velocity data for all four mannans, analyzed using $1 \mathrm{~s}-\mathrm{g} *(s)$ and Extended Fujita approaches, suggest that two of the fungal mannans (FM-1 and FM-3) have a unimodal distribution of
\end{abstract}

Richard B. Gillis

richard.gillis@ nottingham.ac.uk

Trushar R. Patel

trushar.patel@uleth.ca

1 Faculty of Medicine and Health Sciences, Queens Medical Centre, University of Nottingham, Nottingham NG7 2UH, UK

2 National Centre for Macromolecular Hydrodynamics, School of Biosciences, University of Nottingham,

Sutton Bonington LE12 5RD, UK

3 Functional Nanomaterials Lab, King Abdullah University of Science and Technology (KAUST), Thuwal 23955-6900, Kingdom of Saudi Arabia

4 Centre of Glycomics, Institute of Chemistry, Slovak Academy of Sciences, Bratislava 84548, Slovakia

5 Department of Chemistry and Biochemistry, Alberta RNA Research and Training Institute, University of Lethbridge, 4401 University Drive, Lethbridge, AB T1K 3M4, Canada molecular species whereas two others (FM-2 and FM-4) displayed bi-modal and broad distributions, respectively: this demonstrates considerable molecular heterogeneity in these polysaccharides, consistent with previous observations of mannans and polysaccharides in general. These methods not only have applications for the characterization of mannans but for other biopolymers such as polysaccharides, DNA, and proteins (including intrinsically disordered proteins).

Keywords AUC · Extended Fujita approach · MULTISIG · SEDFIT-MSTAR $\cdot$ Sedimentation

\section{Introduction}

Mannans are polysaccharides containing D-mannose, and are found as cell wall components in bacteria, fungi, (moulds and yeast) and plants. Pure mannan is uncommon in plants but it is one of the major components of the yeast cell wall together with glucan, chitin, and protein such as mannoprotein. Mannans have different kinds of structures in various organisms. Figure 1a describes the structure of plant mannan, which has a backbone of linear chains made up of $\beta(1 \rightarrow 4)$-linked Mannopyrosyl (Man $p$ ) residues (Tombs and Harding 1998). Plant mannans occur in the cell walls as heteropolysaccharides, i.e., glucomannans, galactoglucomannans, and galactomannans (Ebringerova et al. 2005). Generally, Candida spp. mannans have an $\alpha(1 \rightarrow 6)$ linked backbone (Fig. 1b) substituted mostly at $O-2$ by different number of linear or branched side oligomannosyl chains composed of $\alpha(1 \rightarrow 2), \alpha(1 \rightarrow 3)$ and $\alpha(1 \rightarrow 6)$ links with or without terminal $\beta(1 \rightarrow 2)$ linkages (Shibata et al. 1992, 1996, 2003, 2007). It was also reported that there are subtle variations in the linkage or number 
Fig. 1 Chemical structure of mannan from a plant sources and $\mathbf{b}$ fungal sources
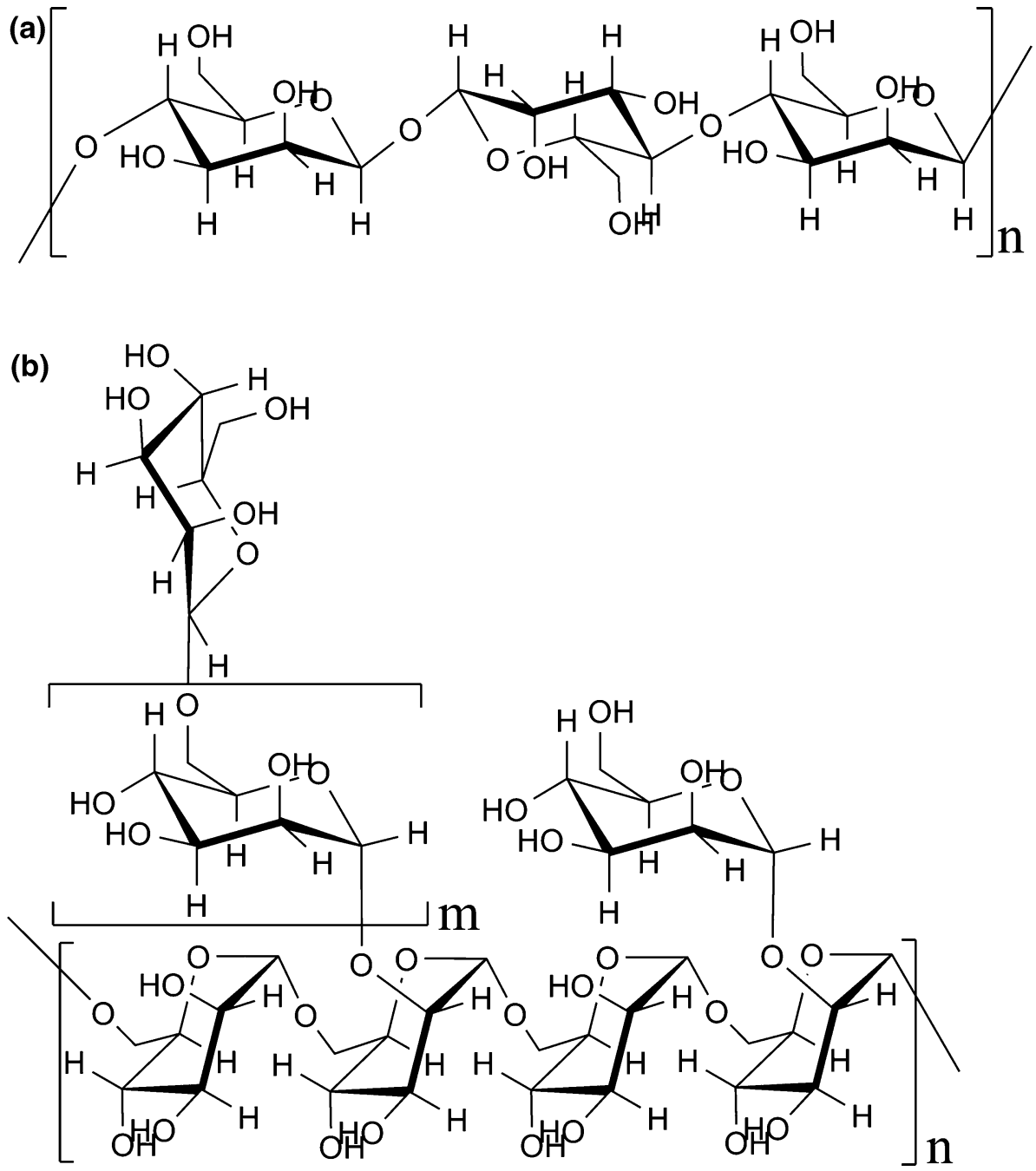

of mannose residues in side chains of Candida spp. mannan molecules (Nelson et al. 1991). C. tropicalis mannan is composed of an $\alpha(1 \rightarrow 6)$-linked backbone substituted only with $\beta(1 \rightarrow 2)$ linked and $\alpha(1 \rightarrow 2)$ linked Man $p$ units without $\alpha(1 \rightarrow 3)$ linked ones observed in $C$. albicans mannan (Kobayashi et al. 1994; Suzuki et al. 1997). C. dubliniensis has been isolated from the HIV-positive individuals in the beginning of 1990 [(Pujol et al. 2004) and references cited therein] and later grouped separately from C. albicans by Sullivan et al. (1995) Mannan from C. dubliniensis (Ližičárová et al. 2005), as well as mannan from C. parapsilosis (Shibata et al. 1995), have very similar structures to that of $C$. albicans.

The anti-tumor activity of polysaccharides was reported by Diller (1947), followed by several other authors who suggested that mannans are potent anticancer agents (Mankowski et al. 1957; Bradner and Clarke 1959; Kamasuka et al. 1968; Oka et al. 1969; Suzuki et al. 1969; Ukai et al. 1983; Peng et al. 2003). It is also critical to characterize these polysaccharides because certain factors, such as polymer length (proportionally linked to molecular weight), can impact their potency, for example their antigenicity (Kabat and Bezer 1958).

This article is focused on the characterization of fungal mannans using analytical ultracentrifugation (AUC) - a well-established, matrix-free method for the determination of molar mass and sedimentation coefficients. In addition, recently presented analysis techniques have been utilized to determine the molar mass distribution of fungal mannans, along with providing distributions of sedimentation coefficients.

\section{Methods}

Four mannan samples, FM-1 (Candida tropicalis CCY 29-7-6), FM-2 (C. dubliniensis CCY 29-177-1), FM-3 (C. albicans CCY 29-3-32), and FM-4 (C. parapsilosis CCY 29-20-1) were prepared from the above-mentioned yeast strains obtained from Culture Collection of Yeast (Institute 
of Chemistry, Bratislava, Slovakia). The mannans were prepared from fresh yeast biomass as described by Bystrický et al. (2003). Under the used strong alkaline conditions, nearly all covalently linked protein was split from the mannoproteins. FM-3 contained $0.4 \%$ nitrogen whereas FM-1, FM-2, and FM-4 only traces.

\section{Sample preparation}

Samples were dissolved in phosphate buffered saline (PBS, $\mathrm{pH} 7.0, I=0.1 \mathrm{M}$ ), made with $0.05 \mathrm{M}$ sodium chloride, and $0.05 \mathrm{M}$ phosphate salts (dibasic sodium and potassium dihydrogen) in screw-capped tubes with constant stirring at low speed. During this period, the temperature was raised to $80.0^{\circ} \mathrm{C}$ for $10 \mathrm{~min}$ to obtain maximum solubility. Stirring continued overnight at room temperature at low speed. Samples were subsequently centrifuged at 10,000 rpm $(11,600 \times g)$ for $15 \mathrm{~min}$ (Beckman L8-55 M Ultracentrifuge). Concentrations of stock solutions were measured using a differential refractometer (Atago DD-5, Jencons Scientific) and a refractive index increment of $0.171 \mathrm{ml} \mathrm{g}^{-1}$ (Mueller et al. 2000).

\section{Molar mass determination using sedimentation equilibrium}

All mannan samples were subjected to sedimentation equilibrium analytical ultracentrifugation (AUC-SE) experiments using the Optima XL-I Analytical Ultracentrifuge (Beckman Instruments, Palo Alto, CA). Double-sector carbon-filled epoxy 12-mm path-length centerpieces, loaded into aluminium housings and sealed with sapphire windows, were used to load solvent $(90 \mu \mathrm{l})$, and sample $(80 \mu \mathrm{l})$ at $1.0 \mathrm{mg} \mathrm{ml}^{-1}$. Cells were loaded into eight-hole titanium rotor (An50Ti) and placed in the centrifuge. Samples were centrifuged at $16,000 \mathrm{rpm}(\sim 20,600 \times g)$ at $(20.0 \pm 0.1){ }^{\circ} \mathrm{C}$. Scans were taken using Rayleigh interference optics once every hour until equilibrium was achieved.

Data analysis was performed using two independent algorithms. SEDFIT-MSTAR (Schuck et al. 2014), which utilizes a smart-smooth method to fit the raw data curve, and $M^{*}$ function (Harding et al. 1992), to provide the weight average of the entire distribution of molar masses:

$M^{*}(r)=\frac{J(r)-J}{k J_{\mathrm{m}}\left(r^{2}-r_{\mathrm{m}}^{2}\right)+2 k \int_{r_{\mathrm{m}}}^{r}(J(r)-J) r \cdot \mathrm{d} r}$

$k=\frac{(1-\bar{v} \rho) \omega^{2}}{2 R T}$

In Eqs. (1) and (2), $r$ is the radial distance from the center of rotation, $r_{\mathrm{m}}$ the corresponding value at the meniscus, $J$ is the concentration in fringe displacement units, and
$J_{\mathrm{m}}$ the corresponding value at the meniscus. $\bar{v}$ is the partial specific volume, $\rho$ is the solvent density, $\omega$ is the angular velocity of the rotor, $R$ is the gas constant and $T$ is the absolute temperature.

MULTISIG was then used to fit the relative concentration proportions of 17 molar masses, logarithmically spaced to achieve a tenfold range, to yield a molar mass distribution (Gillis et al. 2013). The total (fringe) concentration at a set radial position is given by:

$J(r)=\sum_{i=1}^{i=17} J_{\text {ref }} \exp \left\{0.5\left(0.5 k M_{i} 1.15^{(i-1)}\left(r^{2}-r_{\mathrm{m}}^{2}\right)\right)\right\}+E$

where $M_{i}$ is the species molar mass, $J_{\text {ref }}$ is the reference concentration (typically the concentration at the consensus hinge point), and $E$ is the baseline. MULTISIG/RADIUS was used to apply this procedure to radial positions along the column length of solution. Both methods were performed in pro Fit ${ }^{\mathrm{TM}}$ (QuantumSoft, Switzerland).

\section{Sedimentation velocity analysis}

Sedimentation coefficient distributions of mannans were measured using sedimentation velocity (AUC-SV) in the analytical ultracentrifuge. Solvent $(400 \mu \mathrm{l})$ and sample (390 $\mu \mathrm{l}, 1.0 \mathrm{mg} \mathrm{m}^{-1}$ ) were loaded into similarly constructed cells as for AUC-SE. Cells were centrifuged in the Beckman Optima XL-I analytical ultracentrifuge at $40,000 \mathrm{rpm}(\sim 130,000 \times g)$ at $(20.0 \pm 0.1){ }^{\circ} \mathrm{C}$. Data were analyzed using least squares apparent distributions of sedimentation coefficients (1s-g*(s) vs. $s$ ) from SEDFIT (Schuck and Rossmanith 2000), and curve fitting module MULTIG in pro Fit ${ }^{\mathrm{TM}}$ (QuantumSoft, Switzerland). Weight-average sedimentation coefficients $(s)$ for particular components were corrected to standard solvent conditions (density and viscosity of water at $20.0{ }^{\circ} \mathrm{C}$ ) to yield $s_{20, w}$ (S), using SEDNTERP (Laue et al. 1992), and a $\bar{v}$ of $0.625 \mathrm{ml} \mathrm{g}^{-1}$ (Gray and Ballou 1971). The $s_{20, w}$ was measured at a range of concentrations $\left(0.2-2 \mathrm{mg} \mathrm{ml}^{-1}\right)$ for all samples and extrapolation performed for each to zero concentration to obtain $s_{20, w}^{0}$ to eliminate the effects of non-ideality.

\section{Results and discussion}

\section{Molar mass distribution}

Mannan samples, both dialyzed and undialyzed, were probed for their molar masses using sedimentation equilibrium. There was little observable difference in terms of their molar masses between dialyzed and undialyzed 

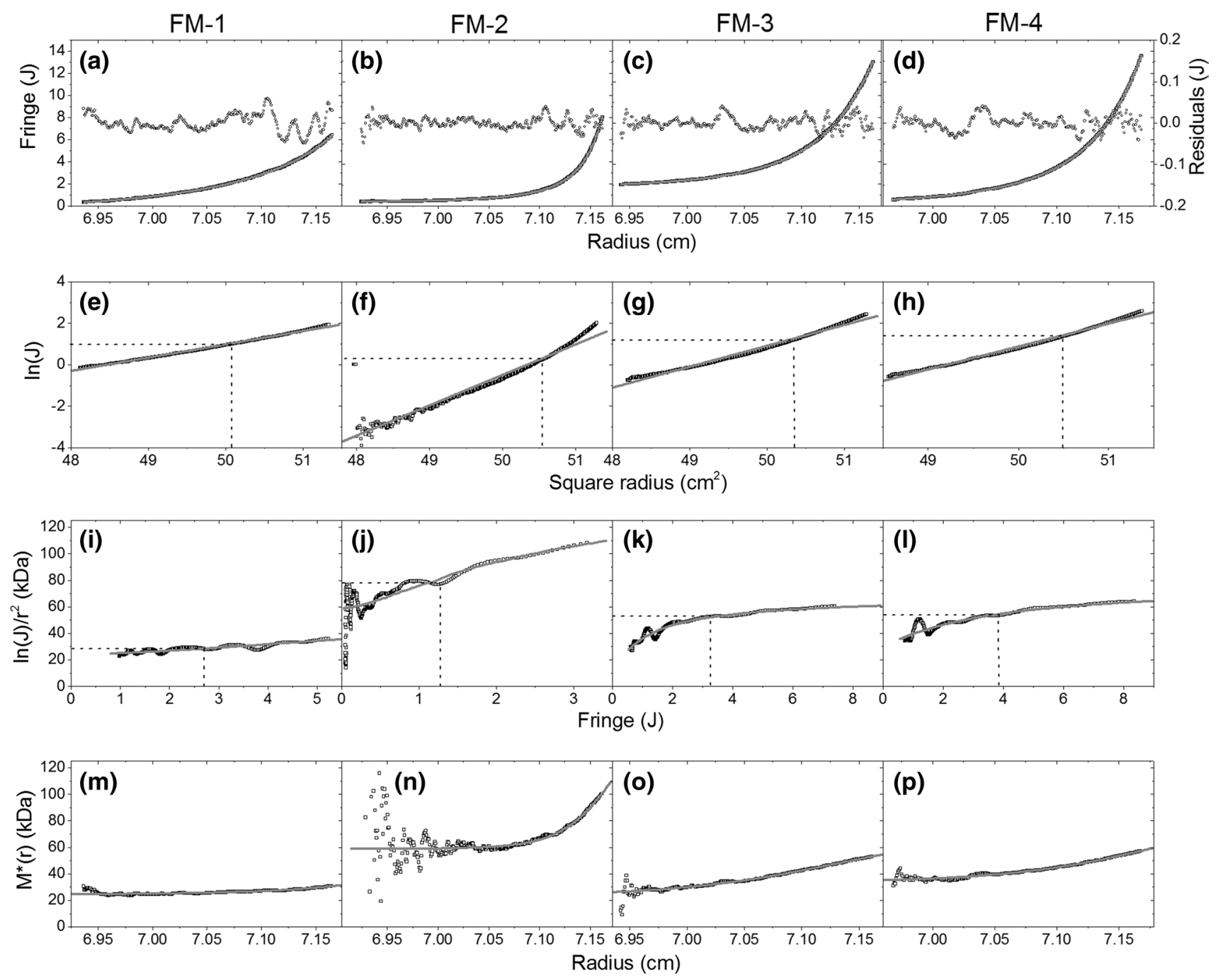

Fig. 2 Output from SEDFIT-MSTAR of FM1-4 (left to right). a-d Fringe displacement (j) vs. radius with residual between raw data (hollow square) and fit (grey line); $\mathbf{e - h}$ natural logarithm of baseline-

samples (data not shown), suggesting the sample was of high purity. Results presented below are from dialyzed samples.

\section{SEDFIT-MSTAR}

SEDFIT-MSTAR fitted the raw fringe displacement data using the smart-smooth analysis (Fig. 2a-d) with results shown in Table 1 . The grey lines represent the result of the fitted parameters. Residuals show no overall trend and do not deviate beyond 0.1 fringes in all samples. The natural logarithm of (baseline-corrected) fringe displacement $(J)$ versus the square of the radius (Fig. 2e-h) shows a nearstraight line but with small positive curvature, particularly in FM-2. This indicates polydispersity. The differential of Fig. 2e-h yields Fig. 2i-1 representing point-average, corrected fringe displacement $J$ vs. square radius; $\mathbf{i}-\mathbf{l}$ differential of e-h yielding apparent molar mass vs. concentration; $\mathbf{m}-\mathbf{p} M^{*}(r)$ algorithm extrapolating to cell base

apparent $M_{w}(r)$ as a function of the concentration $c(r)$ across the cell. Positive slopes suggest polydispersity, especially in FM-2 (Fig. 2j) where values for the $M_{w}(r)$ appear to fall significantly as zero concentration is approached. This result is consistent with results from $1 s-\mathrm{g}^{*}(s)$ analysis suggesting a bimodal distribution; thus the lower radial positions in the cell would contain a high proportion of the low-molar-mass species.

The MSTAR algorithm yielded weight-average molar masses $\left(M_{w}\right)$ ranging from 3.1 to $11.2 \times 10^{4} \mathrm{~g} \mathrm{~mol}^{-1}$ through the extrapolation of the $M^{*}(r)$ to the base of the cell (Fig. $2 \mathrm{~m}-\mathrm{p}$ ) and $z$-average molar masses $\left(M_{z}\right)$ ranging from 4.1 to $14 \times 10^{4} \mathrm{~g} \mathrm{~mol}^{-1}$. The ratio of $M_{z} / M_{w}$ provides the polydispersity index (PDI) ranging from 1.2 to 1.3 , typical for a polydisperse system. Consensus hinge points (CHPthe point at which, during the approach to equilibrium, the 
Table 1 Weight average and $z$-average molar mass estimates from AUC-SE of fungal mannans using SEDFITMSTAR

\begin{tabular}{lllll}
\hline Sample & $\begin{array}{l}\times 10^{-3} w \text {-average molar } \\
\operatorname{mass}^{\mathrm{a}}\left(\mathrm{g} \mathrm{mol}^{-1}\right)\end{array}$ & $\begin{array}{l}\times 10^{-3} w \text {-average molar } \\
\operatorname{mass}^{\mathrm{b}}\left(\mathrm{g} \mathrm{mol}^{-1}\right)\end{array}$ & $\begin{array}{l}\times 10^{-3} z \text {-average molar } \\
\operatorname{mass}^{\mathrm{b}}\left(\mathrm{g} \mathrm{mol}^{-1}\right)\end{array}$ & $\mathrm{PDI}^{\mathrm{b}}(\mathrm{z} / \mathrm{w})$ \\
\hline FM-1 & 30.2 & 31.5 & 41.6 & 1.32 \\
FM-2 & 83.3 & 112 & 140 & 1.25 \\
FM-3 & 56.1 & 54.8 & 64.7 & 1.18 \\
FM-4 & 58.5 & 59.6 & 71.6 & 1.20 \\
\hline
\end{tabular}

Loading concentrations were approximately $1 \mathrm{mg} \mathrm{ml}^{-1}$. Polydispersity index (PDI) measured as a ratio of $z$-average and $w$-average from SEDFIT-MSTAR $c(M)$ fit

${ }^{\text {a }}$ From the Consensus Hinge Point (CHP) method

${ }^{\mathrm{b}}$ From extrapolation of $M^{*}(r)$ to the cell base (Eq. 1, Fig. 2) and SEDFIT-MSTAR fit concentration does not change significantly over time; as well as an indication of the loading concentration of the sample) were measured as an internal check of the rigor of the MSTAR analysis. Hinge points are indicated in Fig. 2 by dashed lines and results are presented, with the other results from MSTAR and $c(M)$ analysis, in Table 1. CHP results do not deviate greatly from the MSTAR and $c(M)$ results, suggesting that non-ideality had no significant impact on the overall analysis (Schuck et al. 2014).

\section{MULTISIG}

MULTISIG approximates the real solute distribution present by a series of 'concentration' coefficients attached to terms in reduced molar mass value, which are logarithmically spaced, thus yielding a distribution $(g(M)$ vs. $M)$ (Gillis et al. 2013). These are presented in Fig. 3. FM-2 and FM-4 both show two peaks, which is consistent with 1s$\mathrm{g} *(s)$ distributions. Number, weight, and $z$-average molar masses, along with polydispersity indices, are shown in Table 2. Estimates are also made in Table 2 for peak molar masses and relative concentrations.

\section{MULTISIG/RADIUS}

Whilst MULTISIG fits 17 discrete species at a selected radial position (in this investigation, the CHP, i.e., the point in the curve where the concentration does not change over the approach to equilibrium), MULTISIG/RADIUS provides this estimate at 20 points along the range of the cell. The baseline was fixed based on ten iterations from the previous MULTISIG analysis. Results yielded number, weight, and z-average reduced molar masses plotted against the concentration range of the cell and a 3D contour plot of $g(M)$ vs. $M$ vs. concentration range (Fig. 4). Number, weight, and $z$-average molar masses produced similar trends to those found from SEDFIT-MSTAR (Fig. 2i-l), which is unsurprising since these plots should provide equivalent information, despite being independently calculated. The contour plot indicates that the twin-peak distributions found in FM-2 and FM-4 are present throughout the cell context with little change in relative strengthwith the exception of FM-2, which has a low proportion of the high- $M_{w}$ species at the top of the cell. This can be explained by the high $g$ force depleting the high- $M_{w}$ component at the meniscus. PDI $(z / w)$ is also consistent to within a reasonable margin of error compared to SEDFITMSTAR values, although a direct comparison is difficult due to the peak-identifying nature of MULTISIG and the whole-solution evaluation from SEDFIT-MSTAR.

Weight averages present themselves directly in-between the two peaks of FM-2 and FM-4, whereas they lie closer to the main peaks present in FM-1 and FM-3; however, they are slightly skewed towards small amounts of smaller (FM-3) or larger (FM-1) material. It is unclear whether these smaller or larger species are indicative of impurities or algorithmic anomalies, however the averages are similar to those found from the independent SEDFIT-MSTAR analysis approach.

\section{Sedimentation coefficient distribution}

The 1s-g*(s) profiles for FM-1, FM-2, FM-3 and FM-4 are shown in Fig. 5, at approximately $2 \mathrm{mg} \mathrm{ml}^{-1}$, as normalized distributions. The profiles indicate that FM-1 and FM-3 yielded a single distribution at $\sim 4 \mathrm{~S}$ with a small degree of larger material in the high-sedimentation range (up to $20 \mathrm{~S}$ ). FM-4 peaks at $\sim 4 \mathrm{~S}$ and has a larger proportion of faster-sedimenting material, particularly at 11 and 14 S. FM-2 yielded a two-peak distribution at $\sim 4$ and $\sim 9$ S.

The concentration for each component was re-calculated based on the initial loading concentration, and amount of mannan fraction present, and used to calculate the $s_{20, w}^{0}$ (S) from $s_{20, w}$. Extrapolations were performed using the reciprocal sedimentation coefficients as shown in Fig. 5 as before (Patel et al. 2006, 2008). The Gralen coefficient, $\left(k_{s}\right)$ was calculated according to Eq. (4) (Rowe 1992) and presented in Table 3 .

$$
\frac{1}{s_{20, w}}=\frac{1}{s_{20, w}^{0}}\left(1+k_{s} c\right)
$$



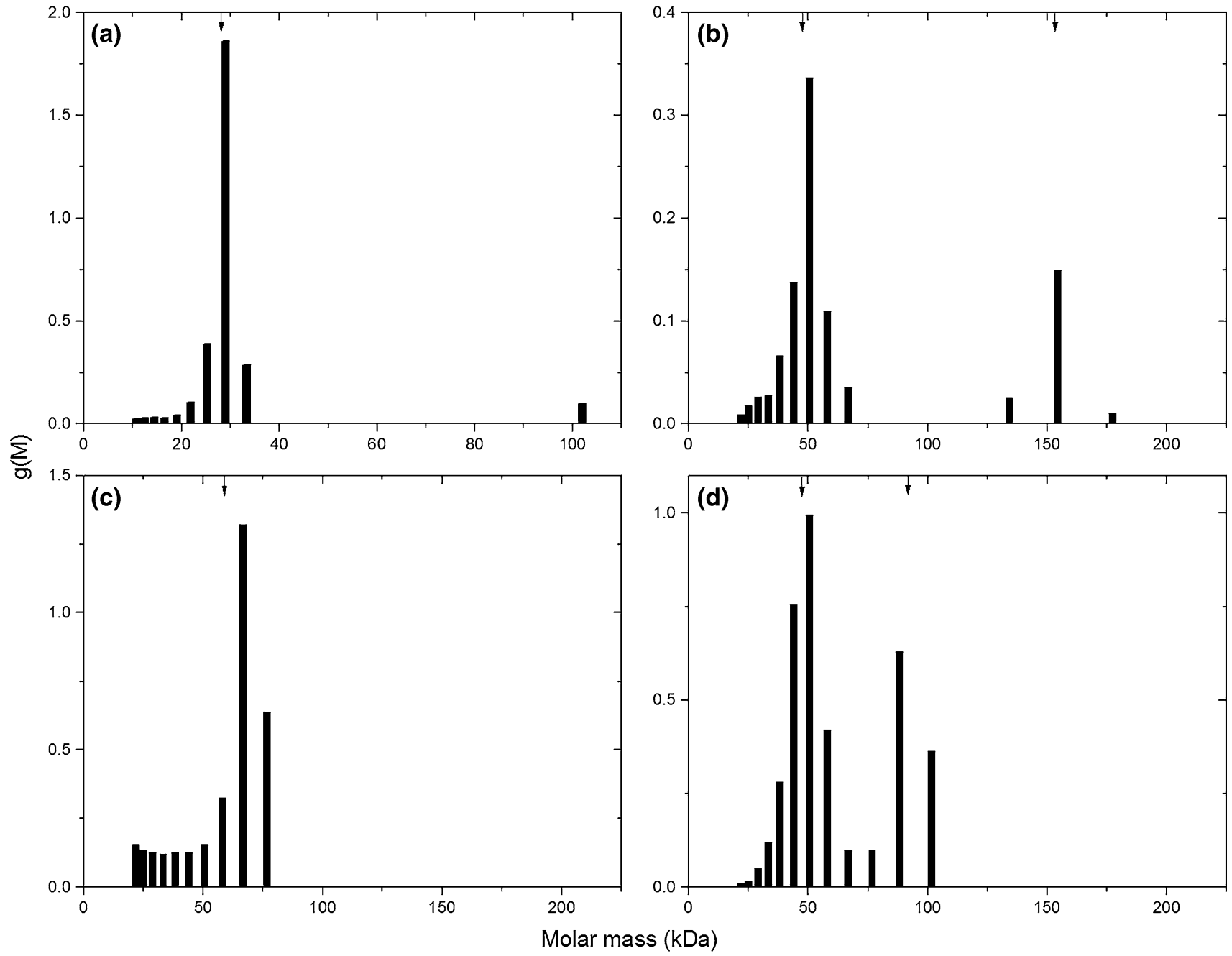

Fig. 3 MULTISIG output of a FM-1; b FM-2; c FM-3; and d FM-4, including 17 discrete molar mass values. Arrows represent weight-average of peak(s)

Table 2 Output from MULTISIG of fungal mannans measured at a loading concentration of $1 \mathrm{mg} \mathrm{ml}^{-1}$

\begin{tabular}{|c|c|c|c|c|c|c|}
\hline Sample & Peak & Fraction $(\%)$ & $\begin{array}{l}\times 10^{-3} n \text {-average molar } \\
\text { mass }\left(\mathrm{g} \mathrm{mol}^{-1}\right)\end{array}$ & $\begin{array}{l}\times 10^{-3} w \text {-average molar } \\
\text { mass }\left(\mathrm{g} \mathrm{mol}^{-1}\right)\end{array}$ & $\begin{array}{l}\times 10^{-3} z \text {-average molar } \\
\text { mass }\left(\mathrm{g} \mathrm{mol}^{-1}\right)\end{array}$ & PDI $(z / w)$ \\
\hline \multirow[t]{2}{*}{ FM-1 } & 1 & 97 & 28.8 & 29.7 & 30.3 & 1.02 \\
\hline & Total & 100 & $29.5( \pm 0.2)$ & $32.3( \pm 0.1)$ & $39.2( \pm 0.0)$ & 1.22 \\
\hline \multirow[t]{3}{*}{ FM-2 } & 1 & 81 & 48.5 & 50.8 & 52.8 & 1.04 \\
\hline & 2 & 19 & 162 & 163 & 163 & 1.00 \\
\hline & Total & 100 & $56.3( \pm 1.0)$ & $67.2( \pm 0.2)$ & $101( \pm 0.0)$ & 1.39 \\
\hline \multirow[t]{2}{*}{ FM-3 } & 1 & 80 & 69.0 & 70.4 & 71.5 & 1.02 \\
\hline & Total & 100 & $54.2( \pm 0.6)$ & $62.3( \pm 0.1)$ & $67.6( \pm 0.0)$ & 1.09 \\
\hline \multirow[t]{3}{*}{ FM-4 } & 1 & 82 & 49.1 & 50.8 & 52.3 & 1.03 \\
\hline & 2 & 28 & 96.9 & 97.6 & 98.3 & 1.01 \\
\hline & Total & 100 & $57.1( \pm 0.2)$ & $64.2( \pm 0.0)$ & $72.3( \pm 0.0)$ & 1.13 \\
\hline
\end{tabular}

Averages $(n, w, z)$ and polydispersity index (PDI, $z / w)$ produced from both the overall distribution ('Total') and from individual peaks. Standard error of the mean represented by parentheses 

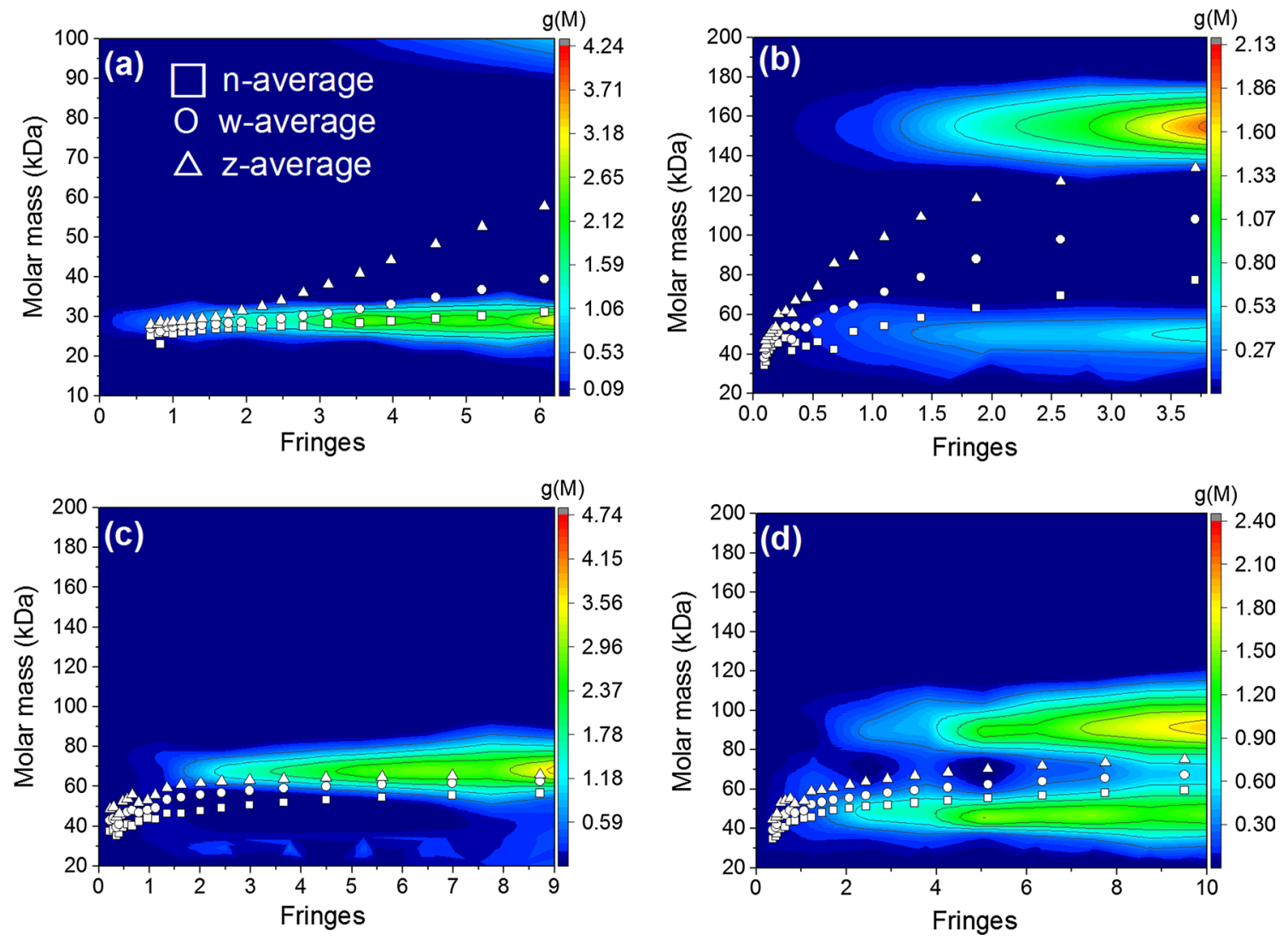

Fig. 4 MULTISIG/RADIUS output of a FM-1; b FM-2; c FM-3; and d FM-4. 17 discrete molar masses along 20 points of the cell. Number (square), weight (circle), and z-average (triangle) molar masses overlaid

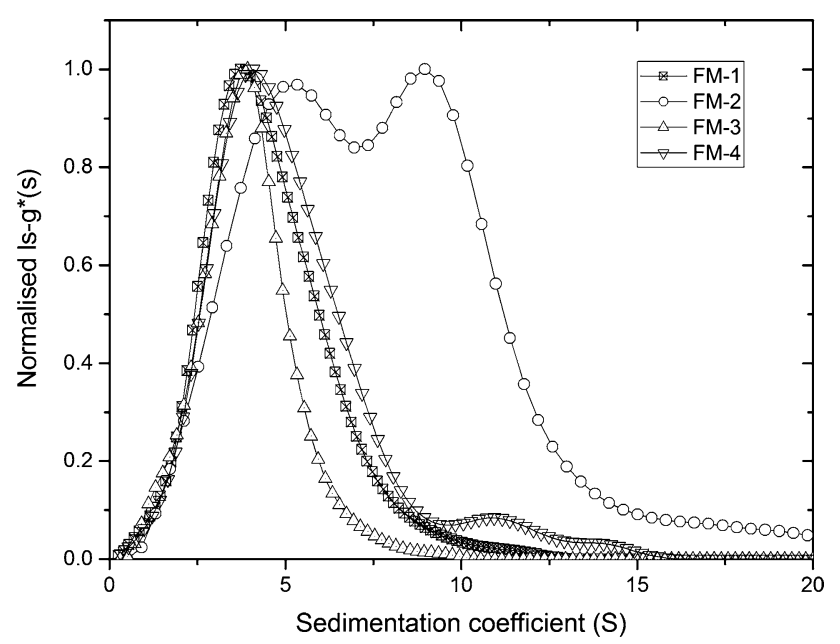

Fig. 5 Normalized $1 s-g *(s)$ vs. sedimentation coefficient of fungal mannans at highest concentration $\left(\sim 2 \mathrm{mg} \mathrm{ml}^{-1}\right)$

Figure 6 shows that FM-1 (a) and FM-3 (c) have one component whereas FM-2 (b) and FM-4 (d) had two different components, based on distributions in Fig. 5. It can be observed that these mannan samples show little
Table 3 Summary of sedimentation velocity analysis of fungal mannan samples, including sedimentation coefficients corrected for solvent conditions and extrapolated to infinite dilution

\begin{tabular}{llcll}
\hline Sample & Peak & Fraction $(\%)$ & $s_{20, w}^{0}(\mathrm{~S})$ & $k_{s}\left(\mathrm{ml} \mathrm{g}^{-1}\right)$ \\
\hline FM-1 & 1 & 100 & $5.6( \pm 0.4)$ & $470( \pm 140)$ \\
FM-2 & 1 & 53 & $5.3( \pm 0.2)$ & $10( \pm 200)$ \\
& 2 & 47 & $9.1( \pm 0.1)$ & $29( \pm 70)$ \\
FM-3 & 1 & 100 & $3.3( \pm 0.1)$ & $137( \pm 65)$ \\
FM-4 & 1 & 92 & $4.3( \pm 0.1)$ & $60( \pm 23)$ \\
& 2 & 8 & $12.8( \pm 0.5)$ & N/D \\
\hline
\end{tabular}

Values in parentheses represent standard error of the mean (S) or regression $\left(k_{s}\right)$

a Peak 2 of FM-4 was averaged (mean), no linear regression was estimated

dependence of concentration on sedimentation coefficients over the small concentration range studied. The second peak from FM-4 showed no definite trend, thus the values were averaged (gradient $=0$ ) to yield $s_{20, w}^{0}$ and no $k_{s}$ estimated because of the very low concentration range.

There is little difference between extrapolated values of native and reciprocal sedimentation coefficients and similar 


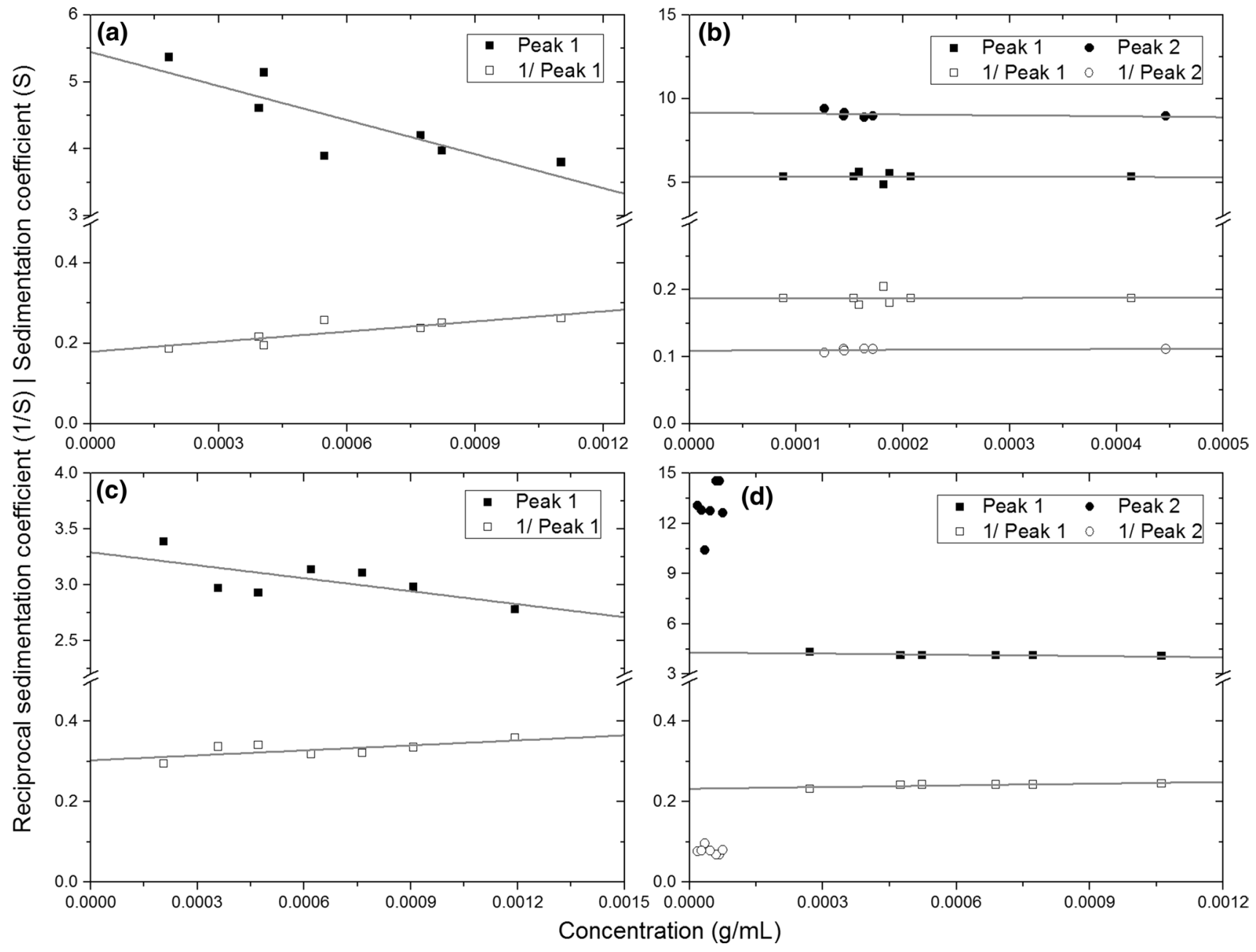

Fig. 6 Extrapolations of integrated peaks from sedimentation velocity measured using linear regression (grey line) of native and reciprocal sedimentation coefficients against concentration of a FM-1; b FM-2; c FM-3; and d FM-4

errors. Values ranged between 3 and $6 \mathrm{~S}$, for the smaller peak. Gralen coefficients differed more between the two methods and generally yielded higher error in the reciprocal regression. The single-peak samples were in the 100 range-118-310 $\mathrm{ml} \mathrm{g}^{-1}$, whereas the two-peak samples were in the tens range- $14-56 \mathrm{ml} \mathrm{g}^{-1}$. This indicates that the single-peak samples have a higher non-ideality than the twopeak systems, however the Gralen coefficients for FM-2 and FM-4 may be affected by the Johnston-Ogston effect (1946).

\section{Molar mass distributions from the extended Fujita approach}

Combining information from AUC-SV (sedimentation coefficients) and AUC-SE (molecular weights) allow for the calculation of power-law scaling factors. The slope of a double logarithmic plot of sedimentation coefficients against molar mass yields the Mark-Houwink-Kuhn-Sakurada (MHKS) sedimentation shape factor $b$ (Eq. 5). $s_{20, w}^{0}=\kappa_{s} M^{b} \quad$ or $\quad M=\left(\frac{s_{20, w}^{0}}{\kappa_{s}}\right)^{1 / b}$

where $\kappa_{s}$ is an intercept constant (not to be confused with the Gralen coefficient, $k_{s}$ ). This factor ranges between $\sim 0.15$ for a 'rod' to $\sim 0.67$ for a 'sphere' and 0.5 is a 'random coil'. A previously published study showed that the MHKS shape factor was 0.43 - this equates to a random coil, but on the 'stiffer' end of the scale (Pavlov et al. 1992). The inset of Fig. 7 shows results from this investigation plotted on a double-logarithmic scale. These data points include individual peaks from FM-2 and FM-4, with peak average molar masses obtained from MULTISIG. The gradient was $0.446( \pm 0.154)$, with a $\kappa_{s}$ of $4.11( \pm 3.38) \times 10^{-2}$, which is well within experimental error of the literature value of 0.43 , corresponding $\kappa_{s}$ of 5.09 $( \pm 0.01) \times 10^{-2}$. The agreement of the $b$ from Pavlov et al. (1992) and the present study justifies the assumption we have made that $M_{w, \text { app }}$ measured at $1.0 \mathrm{mg} \mathrm{ml}^{-1}$ is $\sim M_{w}$. 


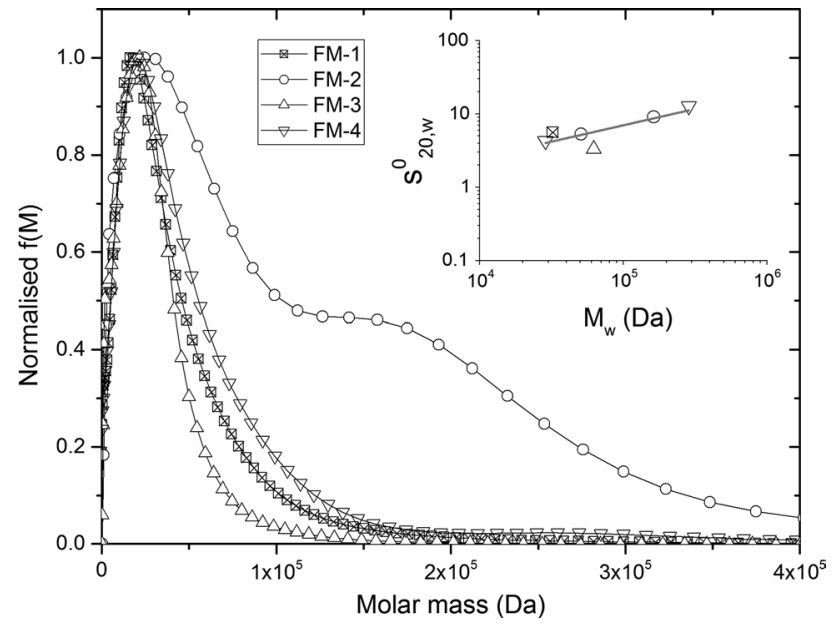

Fig. 7 Extended Fujita approach (normalized $f(M)$ vs. $M$ ) for FM-1, FM-2, FM-3, and FM-4. Inset is Mark-Houwink-Kuhn-Sakurada plot of six peaks from AUC-SV and AUC-SE (MULTISIG)

The extended Fujita approach is a method for yielding molar mass distributions from $\mathrm{g}(s)$ distributions from AUCSV (Harding et al. 2011). The original method assumed that the macromolecule was a random coil, which meant that the sedimentation coefficient was directly proportional to the square root of the molar mass:

$s_{20, w}^{0}=\kappa_{s} M^{0.5}$ or $M=\left(\frac{s_{20, w}^{0}}{\kappa_{s}}\right)^{2}$

Harding et al. (2011) extended this approach to all conformational types using the general power relation (Eq. 6). To transform a distribution of sedimentation coefficients $g(s)$ vs. $s$ to a distribution of molar masses $f(M)$ vs. $M$ the following transformation equations are used:

$f(M)=g(s) \frac{\mathrm{d} s}{\mathrm{~d} M}$

where

$\frac{\mathrm{d} s}{\mathrm{~d} M}=b \kappa_{s}^{1 / b} s^{(b-1) / b}$

In this instance, $1 \mathrm{~s}-\mathrm{g}^{*}(s)$ analysis performed by SEDFIT can be used to substitute $g(s)$ in these equations, with $b$ and $\kappa_{s}$ calculated from the double logarithmic plot of sedimentation coefficient and $M_{w}$. Using Eq. (5) to modify the abscissa and Eqs. (7) and (8) to modify to ordinate, the (normalized) $f(M)$ vs. $M$ plots are shown in Fig. 7. The distributions show a reduction in peak height for FM-2 peak 2 compared to ls-g*(s) from AUC-SV, but correlates more with the $g(M)$ vs. $M$ distribution from MULTISIG AUC-SE. Peak 2 for FM-4 is also reduced, which is less consistent with MULTISIG AUC-SE, which is likely an over-simplification of species present in solution. FM-1, FM-2, and FM-4 all showed overlapping peaks around $2-3 \times 10^{4} \mathrm{~g} \mathrm{~mol}^{-1}$ with broad distributions leading to $\sim 1.5 \times 10^{5} \mathrm{~g} \mathrm{~mol}^{-1}$, consistent with information from AUC-SE.

Polydispersity indices calculated from these distributions were significantly higher than those for MUTLISIG and SEDFIT-MSTAR, with approximately 50-60\% higher estimations for $z / w$. The extended Fujita approach is based on $\kappa_{s}$, which was calculated with a high standard error $\left(4.11( \pm 3.38) \times 10^{-2}, \pm 82 \%\right)$ from Fig. 7 inset. From Eq. (8), it is shown that $\kappa_{s}$ has a large influence on the calculation of $\mathrm{d} s / \mathrm{d} M$ and thus on the spread of the distribution. The high apparent polydispersity from $f(M)$ can therefore be attributed to this high error in the $\kappa_{s}$ value obtained from Fig. 7 inset. Although, in this instance, the extended Fujita approach provided a poor estimation of polydispersity, it did yield accurate estimates for molar mass and heterogeneity.

\section{Non-ideality}

On the subject of non-ideality, concentration dependence was directly measured using AUC-SV to yield $k_{s}$ values, which showed very low non-ideality for all four mannans. AUC-SE did not directly measure concentration dependence, however SEDFIT-MSTAR does provide indications where non-ideality significantly impacts the result. For example, discrepancies between consensus hinge-point and extrapolated $M^{*}(r)$ values, poor $c(M)$ fits and negatively sloping point-average plots would all indicate the presence of significant levels of non-ideality-however they were not observed in the samples analyzed in this investigation. There would also be a poor correlation between the MHKS value obtained from the literature and these mannans. We can therefore say with confidence that there is a negligible effect of non-ideality present, but the values we report are likely to be slight under-estimates from the true values, although not significantly so.

\section{Conclusions}

Four fungal mannan samples were probed for their molar mass and sedimentation coefficients using well-established techniques in the field of polysaccharide characterization.

The two independent analysis techniques used for AUCSE (SEDFIT-MSTAR and MULTISIG) showed very good agreement. The obvious advantage for MULTISIG was the ability to yield molar mass distributions, particularly insightful for FM-2 and FM-4, as well as reliable values for $M_{n}$ and $M_{z}$, but at a cost of processing time (typically, a set of 20 fits can take between 20 and $30 \mathrm{~min}$ ). Compared to 
this, SEDFIT-MSTAR is a much faster analysis method (a fit taking no more than $10 \mathrm{~s}$ ) providing reliable and accurate weight and $z$-average molecular weights but with limited information of molar mass distribution. Combining information from AUC-SE and AUC-SV provided conformation information consistent with previously established results.

These methods (MSTAR, MULTISIG, extended Fujita) have shown a rapid assay for determining the molecular weight distribution of mannans, although they can also be adapted for use with other biopolymer solutions, including polysaccharides, DNA, and proteins-particularly relevant for the characterization of intrinsically disordered proteins. The significance of this assay is the characterization of a polydisperse, heterogeneous biopolymer with significance in various healthcare applications.

Acknowledgments TRP was in receipt of Developing Solutions PhD scholarship.

\section{Compliance with ethical standards}

Conflict of interest The authors declare no competing financial interests.

Open Access This article is distributed under the terms of the Creative Commons Attribution 4.0 International License (http://creativecommons.org/licenses/by/4.0/), which permits unrestricted use, distribution, and reproduction in any medium, provided you give appropriate credit to the original author(s) and the source, provide a link to the Creative Commons license, and indicate if changes were made.

\section{References}

Bradner WT, Clarke DA (1959) Stimulation of host defense against experimental cancer II. Temporal and reversal studies of the Zymosan effect. Cancer Res 19:673-678

Bystrický S, Paulovičová E, Machová E (2003) Candida albicans mannan-protein conjugate as vaccine candidate. Immunol Lett 85:251-255

Diller IC (1947) Degenerative changes induced in tumor cells by Serratia marcescens polysaccharide. Cancer Res 7:605-626

Ebringerova A, Hromadkova Z, Heinze T (2005) Hemicellulose. Adv Polym Sci 186:1-67. doi:10.1007/b136816

Gillis RB, Adams GG, Heinze T et al (2013) MultiSig: a new highprecision approach to the analysis of complex biomolecular systems. Eur Biophys J 42:777-786

Gray GR, Ballou CE (1971) Isolation and characterization of a polysaccharide containing 3-O-methyl-D-mannose from Mycobacterium phlei. J Biol Chem 246:6835-6842

Harding SE, Horton JC, Morgan PJ (1992) MSTAR: a FORTRAN program for the model independent molecular weight analysis of macromolecules using low speed or high speed sedimentation equilibrium. In: Harding SE, Rowe AJ, Horton JC (eds) Analytical ultracentrifugation in biochemistry and polymer science. Royal Society of Chemistry, Cambridge, pp 275-294

Harding SE, Schuck P, Abdelhameed AS et al (2011) Extended Fujita approach to the molecular weight distribution of polysaccharides and other polymeric systems. Methods 54:136-144
Johnston JP, Ogston AG (1946) A boundary anomaly found in the ultracentrifugal sedimentation of mixtures. Trans Faraday Soc 42:789-799

Kabat EA, Bezer AE (1958) The effect of variation in molecular weight on the antigenicity of dextran in man. Arch Biochem Biophys 78:306-318

Kamasuka T, Momoki Y, Sakai S (1968) Antitumor activity of polysaccharide fractions prepared from some strains of Basidiomycetes. Gan 59:443

Kobayashi H, Matsuda K, Ikeda T et al (1994) Structures of cell wall mannans of pathogenic Candida tropicalis IFO 0199 and IFO 1647 yeast strains. Infect Immun 62:615-622

Laue T, Shah B, Ridgeway T, Pelletier S (1992) Computer-aided interpretation of sedimentation data for proteins. In: Harding SE, Rowe AJ, Horton JC (eds) Analytical ultracentrifugation in biochemistry and polymer science. Royal Society of Chemistry, Cambridge, pp 90-125

Ližičárová I, Matulová M, Machová E, Capek P (2005) Mannan from the yeast Candida dubliniensis: isolation and structural characterization. In: 13th Eur. Carbohydr. Symp

Mankowski ZT, Yamashita M, Diller IC (1957) Effect of Candida guilliermondii polysaccharide on transplantable mouse sarcoma 37. Exp Biol Med 96:79-80

Mueller A, Raptis J, Rice PJ et al (2000) The influence of glucan polymer structure and solution conformation on binding to $(1 \rightarrow 3)-\beta$-D-glucan receptors in a human monocyte-like cell line. Glycobiology 10:339-346

Nelson RD, Shibata N, Podzorski RP, Herron MJ (1991) Candida mannan: chemistry, suppression of cell-mediated immunity, and possible mechanisms of action. Clin Microbiol Rev 4:1-19

Oka S, Kumano N, Sato K et al (1969) Antitumor activity of some plant polysaccharides. II. Chemical constituents and antitumor activity of yeast polysaccharide. Gann = Gan 60:287

Patel TR, Picout DR, Ross-Murphy SB, Harding SE (2006) Pressure cell assisted solution characterization of galactomannans. 3. Application of analytical ultracentrifugation techniques. Biomacromolecules 7:3513-3520

Patel TR, Morris GA, Garcia de la Torre J et al (2008) Molecular flexibility of methylcelluloses of differing degree of substitution by combined sedimentation and viscosity analysis. Macromol Biosci 8:1108-1115. doi:10.1002/mabi.200800064

Pavlov GM, Korneeva EV, Michailova NA, Ananyeva EP (1992) Hydrodynamic properties of the fractions of mannan formed by Rhodotorula rubra yeast. Carbohydr Polym 19:243-248

Peng Y, Zhang L, Zeng F, Xu Y (2003) Structure and antitumor activity of extracellular polysaccharides from mycelium. Carbohydr Polym 54:297-303

Pujol C, Daniels KJ, Lockhart SR et al (2004) The closely related species Candida albicans and Candida dubliniensis can mate. Eukaryot Cell 3:1015-1027

Rowe AJ (1992) The concentration dependence of sedimentation. In: Harding SE, Rowe AJ, Horton JC (eds) Analytical ultracentrifugation in biochemistry and polymer science. Royal Society of Chemistry, Cambridge, pp 394-406

Schuck P, Rossmanith P (2000) Determination of the sedimentation coefficient distribution by least-squares boundary modeling. Biopolymers 54:328-341

Schuck P, Gillis RB, Besong TMD et al (2014) SEDFIT-MSTAR: molecular weight and molecular weight distribution analysis of polymers by sedimentation equilibrium in the ultracentrifuge. Analyst 139:79-92. doi:10.1039/C3AN01507F

Shibata N, Arai M, Haga E et al (1992) Structural identification of an epitope of antigenic factor 5 in mannans of Candida albicans NIH B-792 (serotype B) and J-1012 (serotype A) as beta-1, 2-linked oligomannosyl residues. Infect Immun 60:4100-4110 
Shibata N, Ikuta K, Imai T et al (1995) Existence of branched side chains in the cell wall mannan of pathogenic yeast, Candida albicans structure-antigenicity relationship between the cell wall mannans of Candida albicans and Candida parapsilosis. J Biol Chem 270:1113-1122

Shibata N, Akagi R, Hosoya T et al (1996) Existence of novel branched side chains containing-1, 2 and-1, 6 linkages corresponding to antigenic factor 9 in the mannan of Candida guilliermondii. J Biol Chem 271:9259-9266

Shibata N, Kobayashi H, Okawa Y, Suzuki S (2003) Existence of novel $\beta-1,2$ linkage-containing side chain in the mannan of $\mathrm{Can}$ dida lusitaniae, antigenically related to Candida albicans serotype A. Eur J Biochem 270:2565-2575

Shibata N, Suzuki A, Kobayashi H, Okawa Y (2007) Chemical structure of the cell-wall mannan of Candida albicans serotype A and its difference in yeast and hyphal forms. Biochem J 404:365-372

Sullivan DJ, Westerneng TJ, Haynes KA et al (1995) Candida dubliniensis sp. nov.: phenotypic and molecular characterization of a novel species associated with oral candidosis in HIV-infected individuals. Microbiology 141:1507-1521

Suzuki S, Suzuki M, Hatsukaiwa H et al (1969) Antitumor activities of polysaccharides. III. Growth-inhibitory activity of purified mannan and glucan fractions from baker's yeast against sarcoma-180 solid tumor. Gann 60:273-277

Suzuki A, Shibata N, Suzuki M et al (1997) Characterization of $\beta-1$, 2-mannosyltransferase in Candida guilliermondii and its utilization in the synthesis of novel oligosaccharides. J Biol Chem 272:16822-16828

Tombs M, Harding S (1998) An introduction to polysaccharide biotechnology. Taylor and Francis, London

Ukai S, Kiho T, Hara C et al (1983) Polysaccharides in Fungi. XIII. Antitumor activity of the various polysaccharides isolated from Dictyophora indusiata, Ganodeerma japonicum, Cordyceps cicadae, Auricularia auricula-judae, and Auricularia species. Chem Parmaceutical Bull 31:741-744 